\title{
A Review on Various Enhancement Techniques for Mammograms
}

\author{
Jasleen Kaur Chahal \\ M. Tech Student, I\&C Engineering \\ BBSBEC, Fatehgarh Sahib
}

\author{
Navneet Kaur Panag \\ A.P, EE Department \\ BBSBEC, Fatehgarh Sahib
}

\begin{abstract}
Breast cancer has emerged as a health problem is today's world. There are many reasons for the occurrence of breast cancer. Early detection of the breast cancer is important for the proper treatment at the right time. There are many techniques to detect the breast cancer. But the best known is the mammography. Mammography is a low beam $\mathrm{x}$-ray, so for the detection of the cancerous masses contrast enhancement is must in mammography. In this paper we are discussing some of the contrast enhancement techniques used in the mammograms.
\end{abstract}

\section{Keywords}

Mammography, Contrast Enhancement

\section{INTRODUCTION}

Breast cancer is the significant health problem in the world these days. Cancer is a general term that refers to cells that grow and multiply out of control and spread to other parts of the body. According to a survey conducted [1] in year 2012, in India a number of newly detected breast cancer cases were 144,937 and the women died in the same year due to breast cancer were 70,218. When we compare these statics outcome will be that out of the two women with breast cancer one is dying with that. [2] The common risk factors for breast cancer are:

a) Gender: It's not just that women are exposed to breast cancer; males can also have the chances of suffering from disease called breast cancer. But the chances of breast cancer in females are 100 times more as compared to males.

b) Aging: After the age of 55, which is generally the age of menopause there are greater chances of breast cancer.

c) Hereditary: If the person is having cancer in the blood relation then also the chances of breast cancer are more.

d) Birth Control Methods: Women using the temporary birth control measures such as contraceptive pills or DMPA (Depot-medroxyprogesterone acetate) are also at the higher risk of suffering from breast cancer.

e) Personal History: If the person was previously suffering from breast cancer then he/she should opt the regular check up because the again occurrences chances are greater.

f) Race: Race is another factor which also contributes towards the breast cancer. It's found that the women of western nations are more exposed to this disease as compared to the Asian nations. g) Dense breast density: Women with the larger size of their breast as compared to the normal are at higher risk of breast cancer.

h) Being Alcoholic: Another factor that contributes towards the risk factor of breast cancer is being too alcoholic.

It is clear that to cure the breast cancer one should avoid these risk factors and also one should get the proper regular check up. [3] Screening of the breast is best way to know about the cancer at the early stage. Screening means the checking of the breast before the symptoms appear. With this the early detected abnormal tissues can be treated and the cancer can be cured at the early stage. [4] Diagnosing is the next stage to that of the screening, if the results of the screening test found the abnormal tumors in the breast then the person has to go for the diagnosing. [5] The best screening tool is the mammography of the breast. Mammography is a technique in which the patient has to go through the low beam $\mathrm{x}$-ray imaging. There are two types of mammography: filmscreen based mammography and digital mammography. These days digital mammography is widely used as the results obtained in the digital mammography are much clearer. As the mammography is low x-ray beam so it has become a tedious job for the radiologist to detect the cancerous masses. So, to lessen the chances of wrong detection there have been developed many enhancement techniques, some of the enhancement techniques are discussed below.

\section{VARIOUS ENHANCEMENT TECHNIQUES}

\subsection{Histogram Equalization}

The first technique that we are discussing here is Histogram equalization. Mandeep et al [6] Histogram Equalization is a simple technique which is used for the contrast enhancement. The graphical depiction of the image pixels as the function of intensity is the histogram graphical representation. The given image is stretched in the histogram equalization. For the greater contrast enhancement of the image we need a greater stretch in the histogram. The histogram needs to be widened of the corresponding image to get the proper contrast enhancement. There are 256 different intensities for an 8-bit grayscale image; therefore the histogram represents 256 pixel values graphically. Histogram equalization can also be used for the contrast enhancement of the colored images also. For the colored images the histogram equalization can be performed either on the individual colors i.e. red, green and blue or in combination as a $3 \mathrm{D}$ histogram. In the 3D histogram the three axes are representing the red, green, and blue color and the brightness at each point represents the pixel values. The output of the operation is completely dependent that how we carry on the whole process. To confirm the uniform distribution of the intensity of pixel values the gray 
level is remapped using the Histogram Equalization techniques. The dynamic range of the image is flattened and stretched for the overall contrast enhancement of the image. Histogram Equalization is used for the global contrast enhancement of the image.

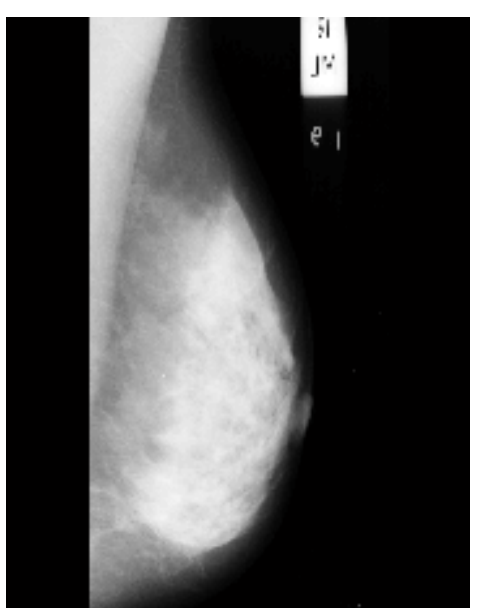

Original Mammographic Image

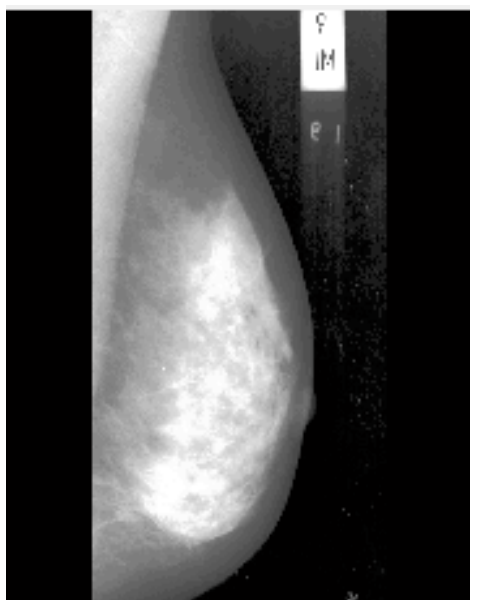

Enhanced Image Using Histogram Equalization

Fig1: Enhancement of image using Histogram Equalization.

\subsection{Enhancement based upon Wavelet Transform and Morphology}

Harish et al [7]For the enhancement of the mammographic image using wavelet transform as well as morphology, the image is divided into two components based upon frequency i.e. lower frequency component and higher frequency component. The frequency splitting is done to achieve larger control degree above dynamic range with Gaussian low pass filter. For the low pass filtered image, the modified mathematical morphology is applied. The low pass filtered image is made to pass through the top hat opening and bottom hat closing. On combining these morphologically contrast enhanced image is obtained. High pass filtered part of the image is constituted of the edge information and noise. To remove the noise and to enhance the edge information, edge enhancement algorithm is implemented. For that from the high frequency component part of the image an arbitrary pixel is selected. Then the function is derived to separate out the noise and edge information. The edge pixels and the noisy pixels are multiplied with the different gain values. The edge enhanced image is obtained with the combination of these values. For obtaining the contrast enhanced image the morphologically enhanced image and the edge enhanced image are added together. To remove the noise element from the image wavelet transform is used. For the wavelet transformation the image is cut into different frequency components, then the resolutions to each component is matched to its scale. The wavelet transformation is constituted of three steps: Wavelet Decomposition, Threshold Detail Coefficients, and Wavelet Reconstruction. After the wavelet decomposition of the image one approximation and six detailed coefficients are obtained. The detailed coefficients are constituted of noisy components, which are de-noised with the proper threshold value. After the de-noising of the proper contrast enhanced image is obtained.

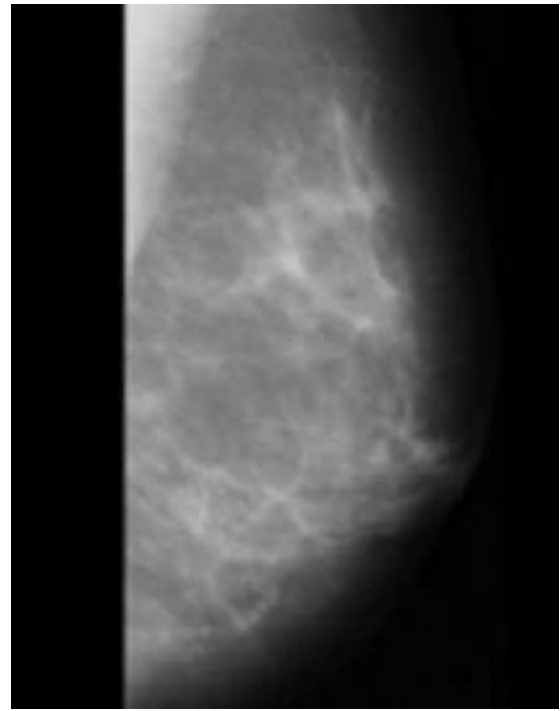

Original Mammographic Image

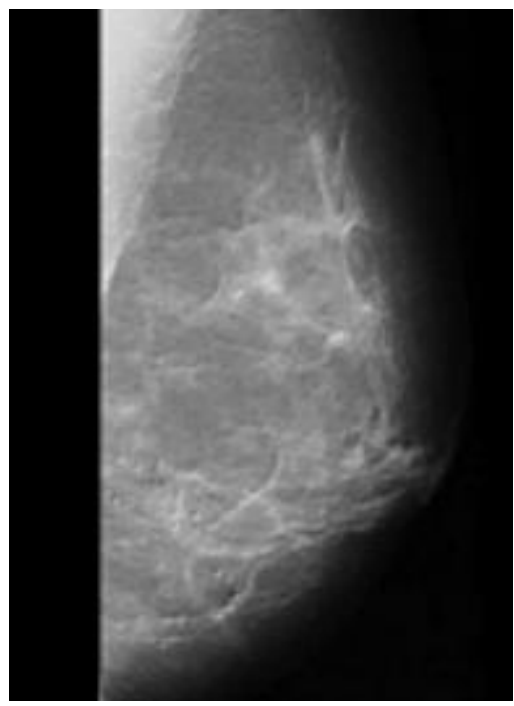

Enhanced Image by Harish et al [7]

Fig 2: Enhancement of mammographic image by Harish et al [7]. 


\subsection{Morphological Operations}

The next technique that we are discussing is considered as a very efficient technique for the contrast enhancement. Bai et al [8] in their paper proposed method called multi-scale transformation. For multi-scale transformation firstly the structuring elements of same shape and increasing sizes are extracted. Dilation and erosion are the multi-scale elements that are important for the proper enhancement of the image. Considering that $f$ represents grayscale image and $B$ represents the structuring element the dilation and erosion are represented with the following equations respectively.

$$
\begin{aligned}
& f \oplus B=\max (g(x-u, y-v)+B(u, v)) \\
& f \ominus B=\min (f(x+u, y+v)-B(u, v))
\end{aligned}
$$

With the help of these dilation and erosion, the multi-scale opening and closing are defined as in the following equations respectively.

$$
\begin{aligned}
& f \circ B=(f \ominus B) \oplus B \\
& f \cdot B=(f \oplus B) \ominus B
\end{aligned}
$$

The White Top Hat (WTH) transformation is applied by subtracting the multi-scale opening from the original image. The Black Top Hat (BTH) transformation is applied by subtracting the original image from the multi-scale closing. The WTH is used to extract the white image region and BTH is used to extract the black image region. The WTH and BTH are defined by the equations as follows:

$$
\begin{aligned}
& \operatorname{WTH}(x, y)=f(x, y)-f \circ B(x, y) \\
& B T H(x, y)=f \bullet B(x, y)-f(x, y)
\end{aligned}
$$

In actual the white and black image regions are present at the different scale values in the image. Considering a sequence of structuring elements having same shape and increasing size at the different scale values: $B_{0}, B_{1} \ldots$ The WTH at the $i$ th scale is defined as:

$$
W T H_{i}=f-f \circ B_{i}
$$

The BTH at the $i$ th scale is defined as:

$$
B T H_{i}=f \cdot B_{i}-f
$$

The white and the black region values are the maximum values at the gray scale levels in the multi-scale image regions and are defined as follows respectively:

$$
\begin{aligned}
f_{w}^{c} & =\max \left\{W T H_{i}\right\} \\
f_{b}^{c} & =\max \left\{B T H_{i}\right\}
\end{aligned}
$$

For the proper enhancement of the image the second to do is the extraction of the detail image regions between neighboring scales. Considering the $i$ th and $(i+1)$ th scale the white top hat region and the black top hat regions are represented as follows respectively:

$$
\begin{gathered}
W T H_{i(i+1)}=W T H_{i+1}-W T H_{i} \\
B T H_{i(i+1)}=B T H_{i+1}-B T H_{i}
\end{gathered}
$$

The final multi-scale black and white detail image regions are defined as follows respectively:

$$
\begin{aligned}
f_{w}^{D} & =\max \left\{W T H_{i(i+1)}\right\} \\
f_{b}^{D} & =\max \left\{B T H_{i(i+1)}\right\}
\end{aligned}
$$

The efficient enhancement of the white image region is obtained by the addition of the white top hat region and white detail image region. On the other hand, the properly enhanced black image region is gained with the addition of the black top hat region and black detail image region and given by equations as follows:

$$
\begin{aligned}
& f_{w}=f_{w}^{c}+f_{w}^{D} \\
& f_{b}=f_{b}^{c}+f_{b}^{D}
\end{aligned}
$$

The final enhanced image is obtained by the addition of the white image region to the original image and subtracting the black image regions and is given by the equation as follows:

$$
f_{E n}=f+f_{w}-f_{b}=f+\left(f_{w}^{c}+f_{w}^{D}\right)-\left(f_{b}^{c}+f_{b}^{D}\right)
$$

Kamra and Jain [9] explained that there are various instrumental techniques for the enhancement which are helpful for the radiologists to detect and diagnose subtle signs in mammograms with more accuracy and reliability. In their paper, they have suggested a morphological based enhancement for the enhancement of distorted architecture consisting of subtle signs. The results in the paper show that the proposed method is showing the better results in subjective as well as objective way.

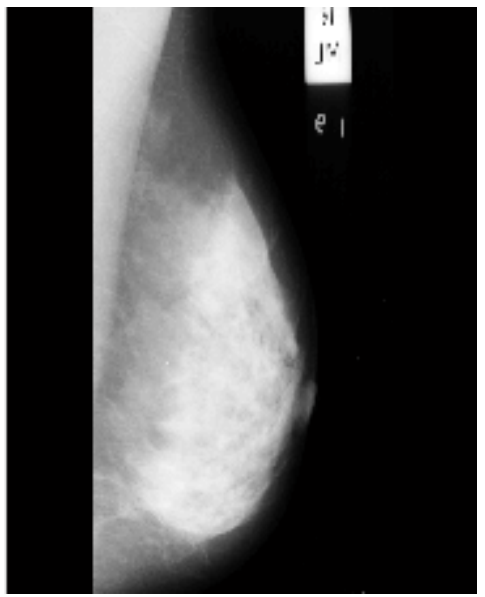

Original Mammographic Image

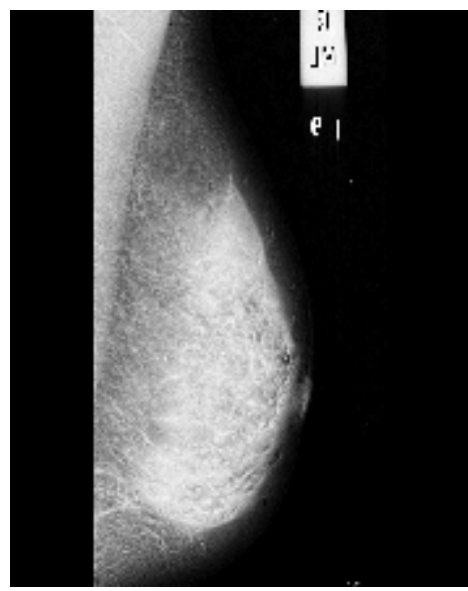

Enhanced Image Using Multi-Scale Morphology

Fig 3: Enhancement of image using multi-scale morphology 


\section{CONCLUSION}

This paper is focused on the different enhancement techniques. From the above discussed techniques it has been clear that a number of enhancement techniques have been developed for the proper contrast enhancement. It has been observed that the histogram equalization technique is used only for the global enhancement of the image; local contrast enhancement with histogram equalization is not possible. Also the histogram equalization fails to remove the noise elements from the image, due to that for histogram equalization is using the images either without noise or with the minor noise contents. Multi-Scale morphology is efficient as easy to implement technique for the proper enhancement of the mammographic images.

\section{REFERENCES}

[1] Statistics of Breast Cancer in India. www.breastcancerindia.net/statistics/stat_global.html

[2] What are the risk factors for breast cancer? www.cancer.org/cancer/breastcancer/detailedguide/breas t-cancer-risk-factors

[3] Breast Cancer Screening $\quad$ (PDQ $\left.{ }^{\circledR}\right)$ www.cancer.gov/types/breast/patient/breast-screeningpdq
[4] Breast Cancer Tests: Screening, Diagnosis, and Monitoring. Available (online) www.breastcancer.org/symptoms/testing/types

[5] Mammography Technique and Types. www.breastcancer.org/symptoms/testing/types/mammog rams/types

[6] Er. Mandeep Kaur, Er. Kiran Jain , Er. Virender Lather, 2013; "Study of Image Enhancement Techniques: A Review", International journal of advanced research in computer science and software engineering, 846-848.

[7] Harish Kumar. N, Amutha. S, Dr. Ramesh Babu D.R., 2012; "Enhancement of Mammographic Images using Morphology and Wavelet Transform", International Journal Computer Technology and Applications Vol 3(1), 192-198.

[8] Xiangzhi Bai, Fugen Zhou, 2010; "Multi-Scale Top-Hat Transform Based Algorithm for Image Enhancement", IEEE ICSP2010 Proceedings, 797-800.

[9] A. Kamra, V.K Jain, "Enhancement of Subtle Signs in Mammograms Using Multi-Scale Morphological Approach", IEEE Point of Health Care Technologies 2013, 54-57. 